\title{
Technique de lutte contre la dégradation de la qualité de l'eau stockée
}

\author{
B.M. Saunier, Président de SAFEGE, \\ J.L. Trancart, Directeur de AQUATECHNIQUE, \\ N. Houel, Directeur de l'Environnement, SAFEGE
}

\section{Introduction}

L'eutrophisation constitue la principale atteinte à la qualité de l'eau des lacs et des retenues. Elle se manifeste par la croissance anarchique d'algues phytoplanctoniques dans la tranche d'eau supérieure (épilimnion) et se trouve fréquemment associée à une désoxygénation de la tranche d'eau inférieure (hypolimnion) à la fois cause et conséquence du phénomène.

Le rôle déterminant des substances nutritives, et notamment du phosphore, dans le développement de l'eutrophisation est maintenant un principe acquis. Ces substances proviennent du bassin versant de la retenue ( charge externe "), mais aussi, et dans certaines conditions physicochimiques, des sédiments reposant sur le fond de la retenue ("charge interne»). Dans ce dernier cas, la présence ou non d'oxygène dissous joue un rôle prépondérant dans les mécanismes de dégradation de la qualité de l'eau. En effet, l'anoxie de la colonne d'eau provoque la réduction du fer et du manganèse complexes à l'état de phosphates dans les sédiments, et le relargage d'importantes quantités de phosphore $\left(15-20 \mathrm{mg} / \mathrm{m}^{2} \times\right.$ jour $)$. Parallèlement, elle provoque d'autres nuisances telles que le blocage du processus de nitrification et l'accumulation d'ammoniaque.

Cette anoxie est engendrée et aggravée par plusieurs facteurs : la stratification thermique des eaux qui se manifeste dans presque toutes les retenues d'une profondeur supérieure à quelques mètres, et la très faible réaération naturelle de ces eaux dites dormantes.

On dispose, pour lutter contre l'eutrophisation, de deux types de stratégies qui sont complémentaires:

— les stratégies à « long terme » qui consistent à contrôler les apports de matières nutritives du bassin versant ;

- les stratégies à " court et moyen terme " qui consistent soit à réduire les effets (par l'épandage d'algicides), soit à empêcher leur manifestation (essentiellement par le blocage du phosphore dans les sédiments).

Quelles que soient les solutions envisagées, leur choix repose sur un diagnostic du plan d'eau qui couvre les aspects suivants :

— diagnostic de l'état du plan d'eau : qualité des eaux et niveau de stratification;
— diagnostic des apports du bassin versant.

Ce diagnostic est fondé sur l'acquisition de données à la fois morphologiques, climatologiques et hydrobiologiques, et sur une connaissance approfondie du terrain. Il peut bénéficier d'outils plus ou moins performants qui permettent par exemple de modéliser simplement le comportement hydrodynamique de la retenue, de simplifier l'étude de terrain ou d'établir des prévisions d'évolution de l'état trophique du plan d'eau.

\section{Diagnostic des plans d'eau}

\subsection{Diagnostic de la qualité des eaux}

L'importance des variations de la qualité de l'eau dans le temps (variations saisonnières) et dans l'espace (variations dans la colonne d'eau) peuvent alourdir considérablement ce diagnostic. Il est important de le limiter à la recherche d'objectifs précis : l'acquisition d'informations de base sur le niveau trophique permettant le cas échéant la détection de signes avant-coureurs de déséquilibre, évaluation de la qualité du milieu en relation avec les utilisations de l'eau (AEP, pêche, loisirs, ...), suivi de l'évolution dans le temps, comparaison avec d'autres plans d'eau.

L'approche préconisée par le CEMAGREF dans ses travaux sur la "diagnose rapide des milieux lacustres » permet de satisfaire ces objectifs. Il s'agit d'une approche limitée aux périodes les plus critiques ou les plus représentatives de l'état du milieu et privilégiant certains descripteurs synthétiques par le choix des points de mesure et des paramètres. Elle concerne à la fois l'eau et les sédiments, compartiments indissociables car interdépendants apportant chacun des informations spécifiques.

La méthode est la suivante :

Compartiment eau : Les interventions ont lieu trois fois par an (fin d'hiver, fin de printemps-été, fin d'automne) de manière à encadrer la période de stratification.

Cinq échantillons sont prélevés sur une verticale au point de profondeur maximale.

Les analyses portent sur la transparence, la température, l'oxygène dissous, le $\mathrm{pH}$, la conductivité, l'azote, le phos- 
phore $\left(\mathrm{PO}_{4}{ }^{3-}\right.$ et Ptot), les bicarbonates, la silice, le fer, le manganèse, la chlorophylle, le carbone organique total.

Elles sont complétées par des analyses des peuplements algaux.

A partir des données recueillies, le calcul d'un indice, appelé l'indice "CERGRENE » car celui-ci est à l'origine de la méthode, permet de classer le lac selon son état trophique.

Sédiments : Ils sont analysés une fois par an et en automne. Des échantillons sont prélevés en plusieurs points distants de quelques mètres autour du point de profondeur maximale pour constituer un échantillon moyen. Les analyses portent sur la phase solide (température, $\mathrm{pH}, \mathrm{rH}$, texture, carbone organique, carbonates, NTK, formes du phosphore) et liquide ( $\mathrm{pH}$, conductivité, azote, phosphore, calcium)

Ces analyses sont ensuite complétées par une étude faunistique qui concerne essentiellement les groupes d'organismes peuplant les zones profondes.

\subsection{Diagnostic du niveau de stratification du plan d'eau}

A partir des profils thermiques mesurés sur le plan d'eau, il est possible de déterminer les gradients de densité formés par les différentes couches de la stratification.

La quantité d'énergie qu'il est nécessaire de mettre en œuvre dans un système de déstratification est déterminée par la somme arithmétique des différences cumulées entre la densité de chaque couche et la densité moyenne de la masse d'eau.

Un facteur correctif est introduit pour tenir compte du rayonnement solaire qui a tendance à reformer cette stratification.

\subsection{Diagnostic des apports du bassin versant}

La réduction de la charge externe en substances nutritives est théoriquement la mesure la plus efficace à long terme. Le choix de moyens techniques et économiques réalistes, pour parvenir à l'objectif de réduction fixé, exige une connaissance minimum de principales sources de pollution et de leurs contributions relatives à la charge totale.

Les estimations les plus précises seront bien sur celles fondées sur des mesures de flux de pollution au droit des différentes sources ponctuelles et à l'exutoire des cours d'eau tributaires. Lorsqu'on ne peut pas disposer de telles mesures, le concept de charge unitaire (quantité de nutriments générée par unité de surface et par unité de temps) peut constituer un moyen rapide d'évaluer les principaux apports en fonction de l'occupation du sol du bassin versant. Il existe de nombreuses publications sur le calcul des charges unitaires pour différentes catégories d'occupation du sol (urbanisations denses ou clairsemées, terres agricoles cultivées, prairies, élevage) [10]. Les coefficients que l'on peut en extraire doivent être manipulés avec précaution, notamment lorsqu'ils dépendent de facteurs susceptibles de largement varier d'une région à l'autre comme le climat ou les pratiques culturales. Dans ce cas, la recherche de données locales (par exemple sur les épandages d'engrais) doit être privilégiée.

C'est le plus souvent un compromis entre mesures et estimations qui présente le meilleur rapport qualité/coût dans l'évaluation des apports de nutriments d'un bassin versant.

Le cas de la retenue de la Visance, résumé ci-après, illustre les démarches possibles.

\section{Le cas de la retenue de la Visance}

L'étude du bassin versant de la retenue s'est concentrée sur le recueil des éléments suivants :

- population agglomérée et population éparse ;

- caractéristiques et performances des réseaux de collecte

et des stations d'épuration d'eaux usées domestiques;

- caractéristiques des rejets industriels, supposées d'après

les données disponibles;

- occupation des terres agricoles et rendements des principales cultures ;

- nombre de têtes de bétail (bovins et porcins);

- les livraisons d'engrais dans la zone, en $\mathrm{kg} / \mathrm{ha}$.

Les apports en phosphore liés aux différentes activités du bassin versant ont été ensuite estimés sur la base de ces éléments et d'hypothèses telles que :

- la quantité de phosphore émise par habitant et par jour (2,5 g/hab/jour) ;

- la quantité de phosphore libérée par an et par kg de bétail, en différenciant bovins et porcins ;

- un coefficient de restitution dans le milieu récepteur des rejets diffus liés aux dispositifs d'assainissement individuel et à l'agriculture (2 à $10 \%$ ).

Ces estimations ont permis de comparer les principales sources en phosphore du bassin versant, qui se répartissent de la manière suivante :

\begin{tabular}{|l|l|c|}
\hline \multicolumn{2}{|l|}{ Rejets } & $\begin{array}{c}\text { Kg P par an restitué } \\
\text { au milieu récepteur }\end{array}$ \\
\hline \multirow{2}{*}{ Rejets domestiques } & Population aggloméré & 480 \\
\cline { 2 - 3 } & Population éparse & $5-26$ \\
\hline Rejets industriels & $113-338$ \\
\hline Rejets liés aux activités agricoles & $143-717$ \\
\hline TOTAL & $741-1561$ \\
\hline
\end{tabular}

Parallèlement, des prélèvements ont été réalisés en plusieurs points du réseau hydrographique du bassin versant de la Visance. Les valeurs mesurées se sont révélées représentatives des estimations réalisées précédemment (flux total 700 à $1700 \mathrm{~kg} \mathrm{P}$ par an selon la période de prélèvement considérée), et confirment l'importance des rejets ponctuels.

\subsection{Etude rapide du bassin versant par imagerie satellite}

La couverture Spot constitue un outil cartographique exploitable dans la connaissance rapide de l'occupation du 


\section{LUTTE CONTRE LA DÉGRADATION DE LA QUALITÉ DE L'EAU STOCKÉE}

sol d'un bassin versant. La classification standard permet de séparer les zones suivantes:

- urbain,

- agricole,

- prairie,

- landes,

- forêt,

- sols nus,

- zones en eau.

Une évaluation de la superficie de ces zones associée au concept de charges unitaires conduit à une estimation grossière des apports en matière de phosphore, suffisante pour apprécier l'existence ou non d'un risque d'eutrophisation et pour guider les investigations complémentaires en matière de réduction des rejets.

\subsection{Les modèles d'eutrophisation}

Ces modèles constituent une méthode simple et facile pour caractériser l'évolution trophique d'une retenue.

Le modèle le plus souvent cité est celui de Wollenweider. Il suppose que la retenue est un réacteur en mélange intégral. Le système s'enrichit en phosphore par les apports des cours d'eau tributaires et du lessivage du bassin versant de la retenue elle-même. Les exportations en phosphore sont déterminées par l'écoulement à l'émissaire de la retenue et par la rétention dans les sédiments (combinaison de la sédimentation et du relargage). Le report sur diagramme des apports en phosphore en $\mathrm{g} / \mathrm{m}^{2} /$ an en fonction du rapport profondeur moyenne/temps de rétention en $\mathrm{m} / \mathrm{an}$ permet de situer une retenue par rapport à son niveau trophique (oligotrophe, mésotrophe, eutrophe).

Ce diagramme a été affiné par plusieurs auteurs, mais toujours selon la même démarche. Malgré les aspects schématiques et simplificateurs de ces modèles, on constate que globalement ils confirment les observations biologiques.

\subsection{Modélisation hydrodynamique}

Le choix du positionnement des installations d'aération diffuse ou hypolimnique est optimum lorsque l'on dispose de données météorologiques et bathymétriques permettant de modéliser l'hydrodynamique du plan d'eau.

Dans la plupart des cas, un modèle bidimensionnel en plan est suffisant pour effectuer un choix opérationnel. En effet, si le lac est peu profond, l'hypothèse d'un milieu uniforme homogène est suffisante. $\mathrm{Si}$ au contraire le lac est stratifié, il n'est pas utile de modéliser le détail de l'évolution de la stratification à partir du moment où l'on sait qu'elle est établie au moins une partie de l'année. Ce qui importe, c'est comme précédemment la description des mouvements d'eau à proximité de la surface.

Classiquement, les modèles bidimensionnels déterminent les champs de courant et les hauteurs d'eau dans tout le domaine modélisé à partir de la résolution des équations de Navier-Stokes.

Le schéma $n^{\circ} 1$ présente les résultats de la modélisation d'un plan d'eau situé à Taiwan. Les lignes de courant engendrées par les vents dominants montrent les secteurs où le brassage naturel est le moins important.

\section{Moyens de lutte contre l'eutrophisation}

\subsection{Stratégies}

Lorsque les éléments du diagnostic sont réunis, bassins versants, qualité des eaux et des sédiments, stratification et circulation des eaux, il est possible d'établir une stratégie de réhabilitation du plan d'eau à partir d'un ensemble de techniques (développées au paragraphe 2.1.2) :

- lutte contre la pollution sur le bassin versant et aménagements hydrauliques destinés à limiter le ruissellement ;

- aménagement des queues de retenues;

- aération ou oxygénation du plan d'eau;

- traitement des sédiments ;

- lutte contre le développement des algues.

La manière de procéder à ces choix techniques s'appuiera sur les principes de base suivants :

\subsubsection{Nature des apports du bassin versant}

Les perspectives de réduction des quantités de phosphore en provenance du bassin versant ne sont en général pas équivalentes. On peut les classer en trois catégories :

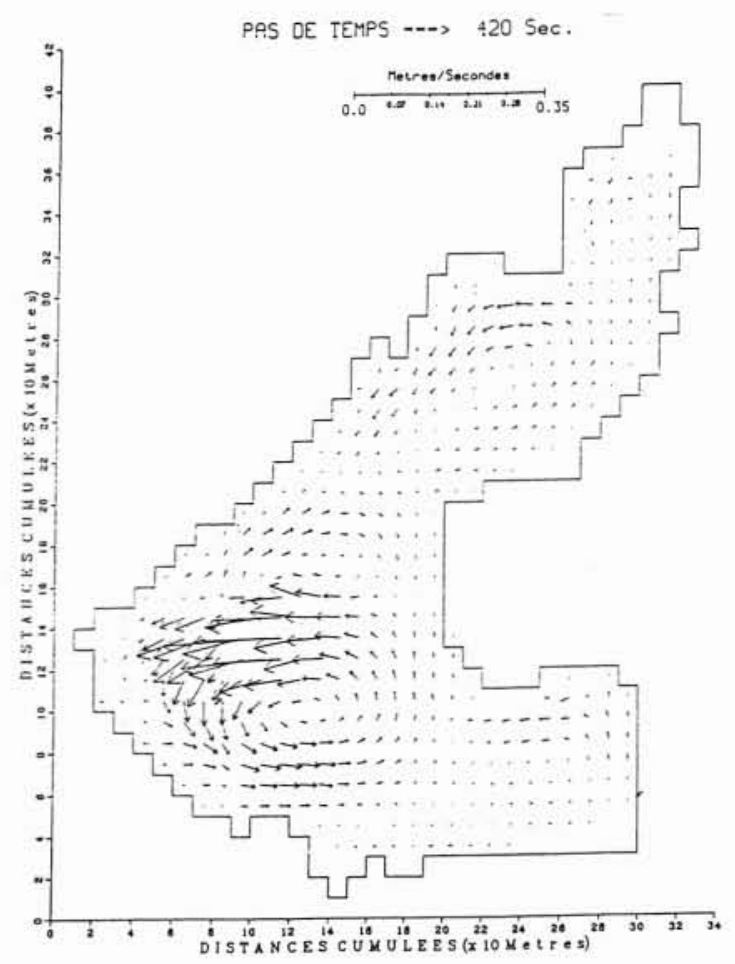

1. 
- Une réduction poussée et rapide est possible : on examinera alors dans ce cas si les quantités de phosphore stockées dans les sédiments (charge interne) sont importantes. Dans l'affirmative, il sera nécessaire de compléter les travaux d'assainissement et d'épuration par la mise en œuvre de techniques de blocage ou d'enlèvement du phosphore contenu dans les sédiments (aération de fond, aération-déstratification, traitement ou curage des sédiments). Le cas typique est celui d'un plan d'eau pollué par des sources localisées et peu nombreuses de phosphore à équiper de traitements spécifiques. Si la charge interne en phosphore est faible, il ne sera pas nécessaire d'envisager des équipements complémentaires.

- Une réduction poussée de la pollution est possible mais à long terme: c'est le cas de sources de pollution en majorités ponctuelles (industries, collectivités) qu'il faut équiper de traitements de déphosphatation mais dont les systèmes de collecte doivent être étendus ou rénovés en profondeur. Suivant l'importance respective de la charge interne et des prévisions de réduction de la charge externe, on mettra en place des équipements spécifiques : aération, traitement des sédiments (pour la charge interne), aménagement des queues de retenues (pour la charge externe résiduelle). Ces mesures permettent de soutenir la qualité du plan d'eau jusqu'à ce que les investissements engagés sur le bassin versant soient pleinement efficaces.

- Seule une réduction partielle est envisageable : cela sera souvent le cas de bassins versants agricoles (élevage) ou dont l'habitat est dispersé et pour lesquels les mesures à prendre concernent une très grande quantité de petites sources ponctuelles. Il peut exister également des secteurs où il existe des apports naturels de phosphore. Dans ces configurations, il est probable que la réduction des apports externes ne puisse atteindre à moyen terme des niveaux suffisamment bas. On envisagera alors de recourir à des procédés de soutien (aération par exemple) et il sera nécessaire d'effectuer régulièrement des traitements algicides.

\subsubsection{Types d'usages de l'eau}

Suivant les usages de l'eau et les degrés d'urgence présentés ci-dessus, il sera tenu compte, dans le choix des techniques, de contraintes particulières. Ainsi, pour préserver une zone de baignade dans l'attente d'effets à moyen terme de la réduction des apports, il est possible d'équiper les secteurs les plus fréquentés de barrières à bulles permettant d'éviter la pénétration de résidus végétaux, d'améliorer la transparence et de lutter contre les mauvaises odeurs. Dans certains cas, l'injection de désinfectants peut être couplée au système de bullage.

Dans le cas des plans d'eau destinés à l'eau potable, on cherchera plus particulièrement à protéger la zone de la prise d'eau des relargages de fer, de manganèse et d'ammoniaque qui perturbent les traitements de potabilisation. Pour ce faire, des systèmes d'aération localisés sont à préconiser. Une action particulière contre le développement de cyanophycées, dont la présence dégrade la qualité organoleptique de l'eau distribuée, peut également être nécessaire : algicides, agitation de l'eau.
Enfin, dans le cas de plans d'eau dont il faut préserver le cheptel piscicole des conséquences de déficit en oxygène, il sera nécessaire d'équiper de grandes surfaces du plan d'eau d'un système d'aération qui ne fonctionnera que pendant de courtes périodes critiques.

\subsubsection{Caractéristiques physiques du plan d'eau}

Un certain nombre de caractéristiques physiques du plan d'eau doivent être prises en considération avant d'orienter le choix et l'emplacement de techniques de réhabilitation.

La stratification: Lorsqu'une aération du plan d'eau est nécessaire, il est important de se déterminer entre une aération de fond ou une aération déstratification. La stabilité et le gradient de température de la stratification ainsi que l'énergie nécessaire pour la briser doivent être connus. Dans le cas de lacs profonds $(>15 \mathrm{~m}$ ), et stratifiés de manière stable, il est préférable, pour minimiser les dépenses d'énergie, de s'orienter vers une aération gyrothermique. En revanche, dans des lacs peu profonds et à faible gradient de température, l'aération déstratification constituera un choix optimal.

La morphologie: Outre la profondeur, la forme du plan d'eau peut offrir la possibilité d'installer un aménagement particulier de queue de retenue. Par ailleurs, une modélisation fondée sur la morphologie du plan d'eau et l'influence du vent permettra de mieux connaître les régimes de brassage naturels de l'eau et d'orienter le choix du type et de la position des équipements d'aération.

\subsubsection{Considérations économiques}

En l'absence de normes sur ce que doit être la qualité d'un plan d'eau, l'examen de l'opportunité de telle ou telle aspiration de reconquête de la qualité des eaux d'une retenue ou d'un lac pourra s'appuyer sur trois types de considérations économiques :

- La comparaison finale du coût de mesures de dépollution sur le bassin versant et d'équipements de traitement in situ. Celui d'une usine de traitement dans le cas de l'eau potable.

- La comparaison du coût de la dépollution (réseaux, station d'épuration) avec celui de mesures d'accompagnement permettant de garantir une réhabilitation complète du plan d'eau.

- L'examen de la durée d'amortissement d'équipement de soutien de la qualité avec la prévision de la durée de mise en place définitive des opérations de dépollution sur le bassin versant.

\subsection{Revue des techniques}

\subsubsection{L'aération et l'oxygénation de l'hypolimnion}

Lorsqu'il existe une stratification marquée, les perturbations du milieu sont minimisées en procédant au maintien des conditions aérobies dans les eaux profondes par injection soit d'oxygène pur, soit d'air comprimé. Rappelons que l'augmentation de la teneur en oxygène à l'interface 
eau-sédiment crée des conditions rédox qui évitent le relargage des formes réduites du fer, du manganèse et de l'azote et évitent la libération de phosphore sous forme de complexes solubles.

L'utilisation de l'oxygène pur : Cette solution a été retenue dans le cas de la retenue de la Meaugeon qui alimente la ville de St-Brieuc en eau potable (R); son coût d'exploitation est cependant élevé en raison de l'utilisation d'oxygène pur.

L'utilisation de l'air comprimé : L'aération d'hypolimnion par injection d'air comprimé est présentée, quant à son principe, en figure 2. Elle ne requiert pour son fonctionnement que l'alimentation électrique d'un compresseur. L'eau de fond aspirée par air-lift est oxygénée sous pression par contact avec les microbulles diffusant dans l'aérateur; après réoxygénation dans celui-ci, elle est ensuite réinjectée horizontalement par des tuyères à la base de l'aérateur. Le rendement énergétique moyen de l'aération d'hypolimnion, en fonction de la profondeur, de la température de l'eau et de son niveau de saturation, dépasse $1 \mathrm{~kg}$ d'oxygène transféré par $\mathrm{kWh}$ fourni. La réalisation des équipements immergés (alimentations, aérateurs) en matériaux exclusivement synthétiques (évitant le risque de corrosion), l'absence de toute pièce mobile, constituent des avantages importants pour l'exploitation des installations.

Le procédé LIMNO fait l'objet à ce jour de 68 installations dans le monde, initialement réalisées en fibre de verre rigide et plus récemment en matériau souple. La figure 3 présente les profils thermiques et d'oxygène dissous du lac Lagunitas (Californie, U.S.A.) traité depuis 1988 avec une unité LIMNO de $10 \mathrm{~m}$ de hauteur. Il ressort de l'observation des résultats :

- la très forte augmentation des teneurs en oxygène dissous dans l'hypolimnion après la mise en place du traitement ;

- le maintien de la stratification thermique de la colonne d'eau.

Les suivis de ce type de traitement montrent la réduction importante des teneurs en phosphore dissous, ammoniaque, fer et manganèse [1]. La figure 4 présente les coûts de traitement de l'eau potable sur l'unité de traitement de Norwalk (Connecticut, U.S.A.) dont le réservoir d'eau brute, le City Lake, est traité par aération d'hypolimnion pendant la majeure partie de l'année [2]. L'économie induite par le traitement sur les consommations de réactifs de l'usine d'eau potable se chiffre dans ce cas à $15 \%$. Les coûts de traitement par aération d'hypolimnion rapportés au $\mathrm{m}^{3}$ d'eau traitée (cas d'un réservoir d'A.E.P.) dépendent essentiellement du ratio (volume de la zone traitée/production de l'unité de potabilisation). Ce coût d'exploitation (énergie, maintenance) se situe en moyenne entre 0,01 et $0,03 \mathrm{~F} \mathrm{HT} / \mathrm{m}^{3}$ d'eau traitée.

\subsubsection{La déstratification ou aération diffuse}

Pour des plans d'eau dont la profondeur ne permet pas le développement d'une stratification thermique nette en période chaude, l'aération diffuse constitue une bonne

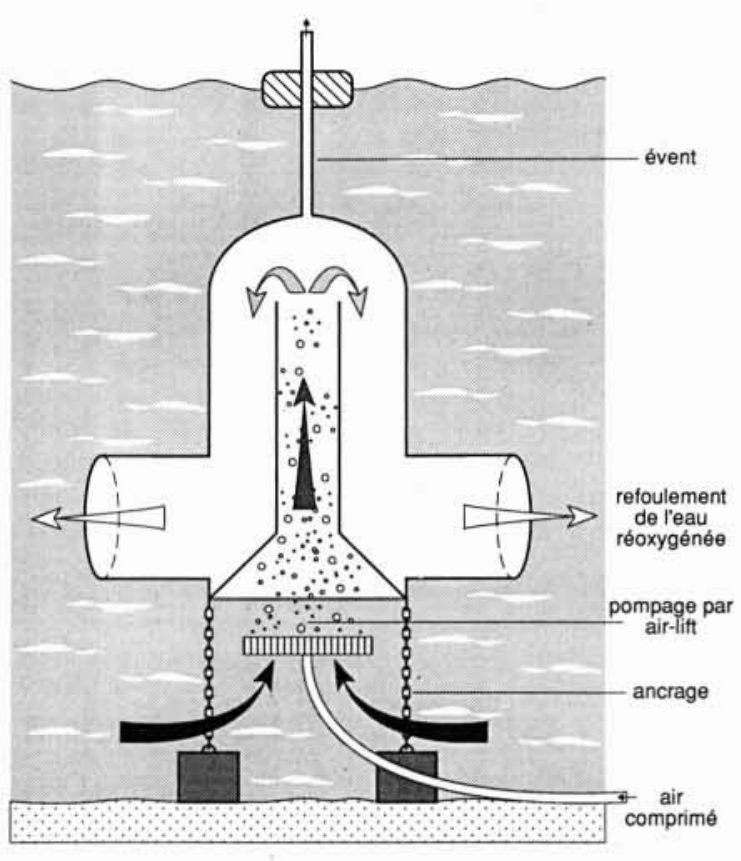

2. Principe de fonctionnement de l'aérateur d'hypolimion LIMNO (modèle breveté).

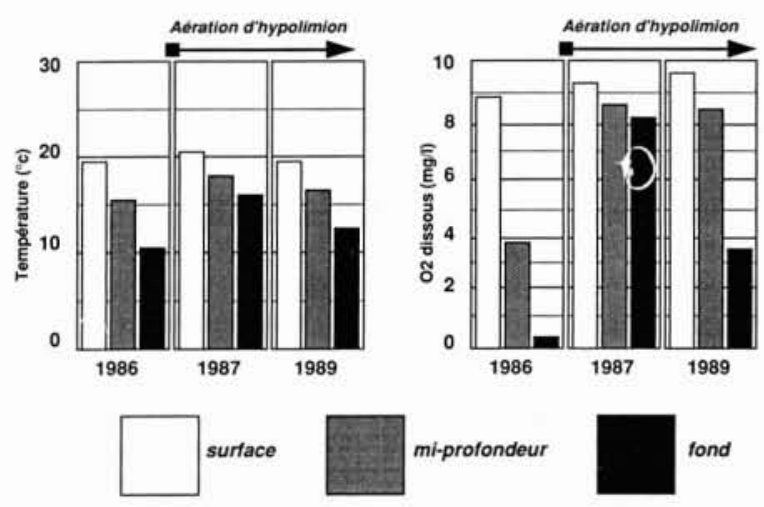

3. Lac Lagunitas - température et oxygène dissous.

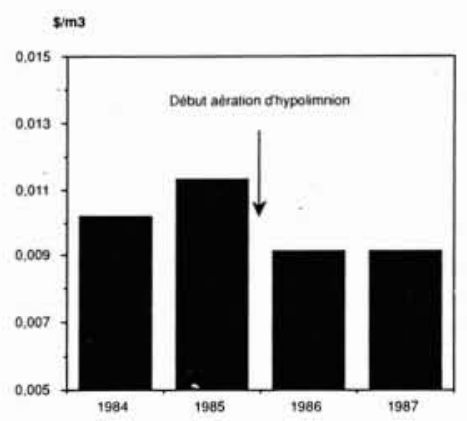

4. Coûts unitaires moyens de traitement de l'eau du City Lake. 


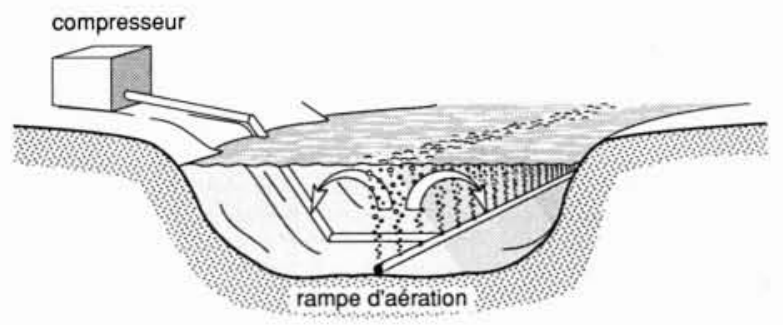

5. Schéma de principe de fonctionnement de l'aération diffuse.

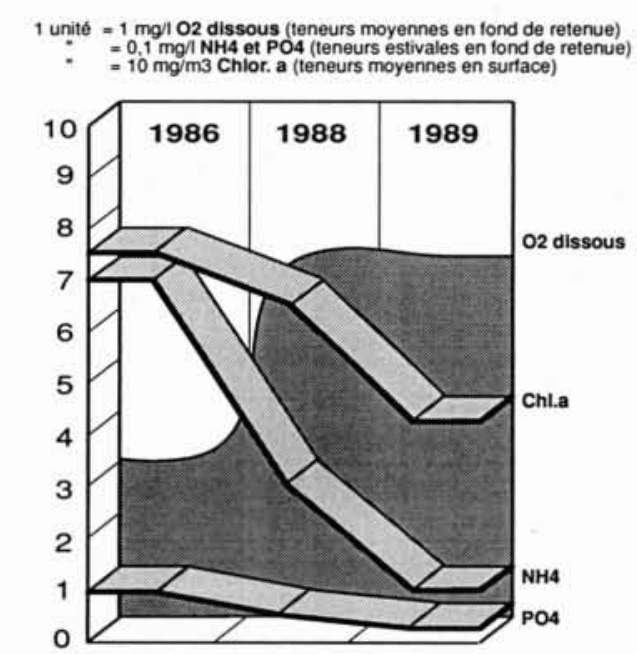

6. Qualité estivale des eaux de la retenue de la Visance (mise en service des traitements en juin 1981).

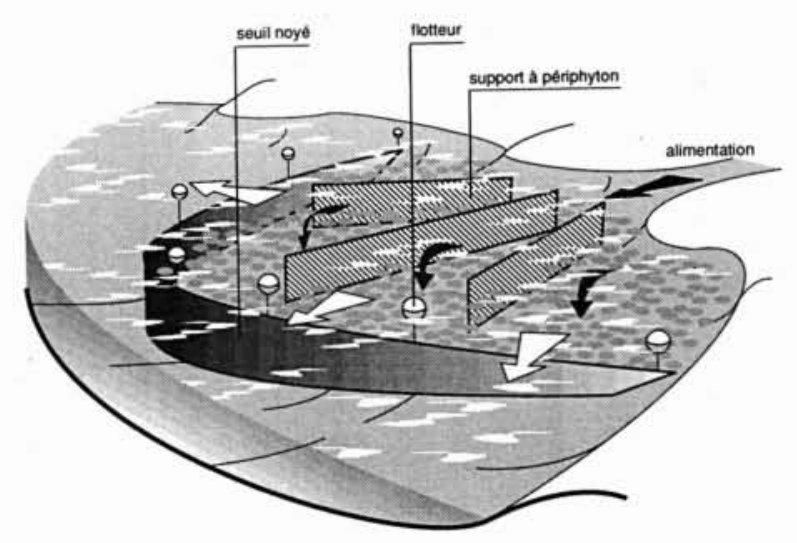

7. Principes de mise en auvre du traitement biologique de queue de retenue (procédé breveté Pred-Bassin). solution pour lutter contre le développement de l'anoxie. L'air comprimé, fourni par un compresseur situé sur la rive, alimente des rampes en polyéthylène flottant immédiatement au-dessus du fond (schéma en fig. 5). L'oxygénation de la colonne d'eau est obtenue :

- par transfert à la colonne d'eau d'une partie de l'oxygène de l'air injecté ;

- par réaération superficielle des eaux de fond mises en convection au droit de l'injection d'air.

Malgré un rendement énergétique direct légèrement moins bon de ce procédé comparé à l'aération d'hypolimnion, les puissances installées restent tout à fait modestes. Un grand nombre d'installations de déstratification ont été installées sur des plans d'eau peu profonds de différents types en Europe et aux U.S.A. notamment ; en France, on citera les cas de la retenue du Puy-Terrier sur le Cebron (Deux-Sèvres) [3] en date de 1986, et plus récemment, de la retenue de la Visance, alimentant la ville de Flers (Orne) en eau potable. LeVET et MARTIN [3] citent les effets suivants du traitement mis en œuvre sur la retenue du Puy-Terrier : élévation de la transparence de l'eau, disparition des odeurs désagréables à l'aval du barrage et forte élévation des teneurs en oxygène dissous en fond de retenue, réduction des teneurs en ammoniaque, fer et manganèse dans l'eau brute, réduction des teneurs en matières organiques et des doses de floculants nécessaires à l'usine de traitement.

La figure 6 montre l'évolution de la qualité des eaux de fond de la retenue de la Visance pour les années 1986, 1988 et 1989, le traitement de déstratification réalisé par SAFEGE (auquel est adjointe une déphosphatation sur la principale station d'épuration du bassin versant) ayant commencé au début de l'été 1988.

En matière algale, la déstratification permet de réduire la proportion des cyanophycées qui se développent préférentiellement à la base de l'épilimnion [1]. C'est le cas de la retenue de la Visance, où l'on observe le passage d'une population à dominance de cyanophycées (Microcytis sp.) [4] à des chlorophycées. L'intérêt de cette évolution est la réduction importante des problèmes de traitement et de mauvais goût spécifiquement liés aux cyanophycées.

Les coûts de traitement par aération diffuse dans le cas de cette retenue se sont élevés à $0,075 \mathrm{~F}$ HT par $\mathrm{m}^{3}$ d'eau traitée (valeur 1989). Dans le même temps, les coûts de réactifs à l'usine de potabilisation étaient réduits de $30 \%$, rapporté au $\mathrm{m}^{3}$ d'eau traitée, les volumes filtrés entre deux cycles de lavage s'accroissaient de $37 \%$ en moyenne, et le traitement de la retenue au sulfate de cuivre a été supprimé.

\subsubsection{Traitement biologique de queue de retenue}

Les principes essentiels du traitement biologique de queue de retenue sont les suivants :

- accroissement estival du temps de séjour hydraulique ; - réchauffement et éclairement important de la masse d'eau en zone d'alimentation;

- élévation de la part du phosphore assimilable apportée par les tributaires. 


\section{LUTTE CONTRE LA DÉGRADATION DE LA QUALITÉ DE L'EAU STOCKÉE}

Ces processus ont conduit à aménager, souvent sur des ouvrages existants, des dispositifs visant à améliorer ou créer les conditions de lagunage des eaux d'alimentation dans la partie amont de la retenue, avec les effets suivants :

- homogénéisation des temps de séjour hydraulique dans la zone de traitement, favorisant l'adsorption sur les sédiments des phosphates en excès dans les tributaires;

- assimilation biologique du phosphore soluble (d'où un abattement important dans le dispositif des teneurs en $\left.\mathrm{P}_{-} \mathrm{PO}_{4}\right)$, et décantation d'une partie de la biomasse à l'amont du seuil, à une profondeur minimisant les risques d'apparition de l'anoxie.

Lorsqu'il n'existe pas de bassin correctement dimensionné dans la partie amont du plan d'eau à traiter, une solution intéressante consiste dans la mise en place d'une membrane synthétique souple immergée; la circulation amont-aval est assurée par la lame d'eau supérieure, sans perte de charge au droit de celle-ci (fig. 7). Ce dispositif peut s'adapter aux variations éventuelles de la cote du plan d'eau. Le seuil peut si nécessaire être déplacé vers l'aval en cours de saison, voire remisé en période hivernale. La maintenance du dispositif est extrêmement simple. L'installation du seuil peut être combinée à celle de supports synthétiques fixant le périphyton (microalgues fixées) à l'amont du seuil.

L'efficacité de tels traitements a notamment été examinée par Fiala et VASATA [5], BENNDORF et Putz [6] et VYMAZAL [7], ce dernier pour les supports à périphyton. Dans le cas du réservoir de Jesenice (Tchécoslovaquie), les premiers auteurs mettent en évidence entre l'amont et l'aval du dispositif une réduction de $90 \%$ du phosphore soluble, de 65 à $70 \%$ du phosphore total et de 30 à $40 \%$ sur l'azote total (fig. 8) ; BENNDORF et PuTz [6] citent une élimination théorique maximale de $60 \%$ de $\mathrm{P}_{-} \mathrm{PO}_{4}$ en fonction du temps de séjour. VYMAZAL [7] fait état d'abattements mesurés de $70 \%$ sur $\mathrm{P}_{-} \mathrm{PO}_{4}$ sur supports à périphyton.

\subsubsection{L'oxydation in situ des sédiments de lacs et retenues par injection de nitrate de calcium}

On a vu précédemment que le caractère réducteur des sédiments constituait une des causes de dégradation de la qualité des eaux de plans d'eau, avec pour effets :

- la consommation de l'oxygène dissous de la colonne d'eau ;

- le relargage des phosphates par suite de la réduction du fer à l'état ferreux.

Ces processus hypothèquent le rétablissement de la qualité des plans d'eau à l'issue de la réduction des charges externes de nutriments apportées à celui-ci. Devant le coût généralement prohibitif de l'extraction des sédiments, il peut être intéressant d'opérer l'oxydation sur place de la couche superficielle des sédiments, opération réalisable à l'aide de nitrate de calcium. La réaction globale est décrite par l'équation suivante :

$$
\begin{aligned}
5 \mathrm{C}_{6} \mathrm{H}_{12} \mathrm{O}_{6}+24 \mathrm{H}^{+} & +24 \mathrm{NO}_{3}{ }^{-} \rightarrow 12 \mathrm{~N}_{2}+42 \mathrm{H}_{2} \mathrm{O} \\
+ & 30 \mathrm{CO}_{2} .
\end{aligned}
$$

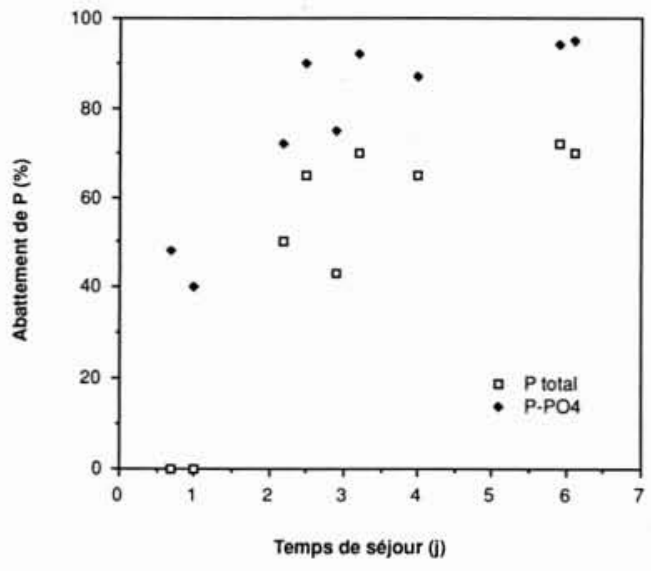

8. Abattement de P par traitement de queue de retenue.

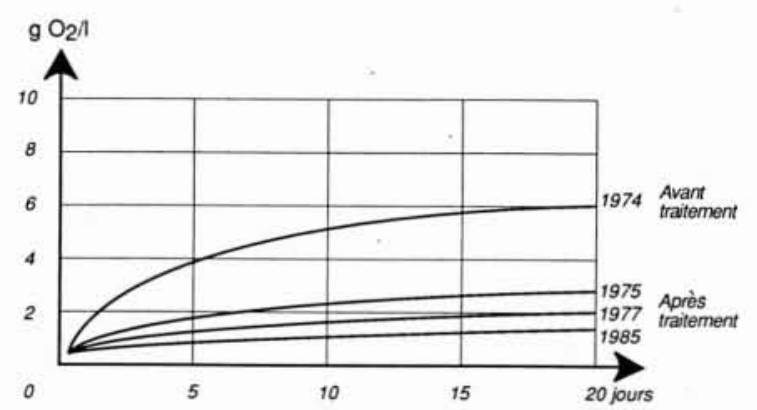

9. Consommation en oxygène des sédiments du lac Tillesjoen avant et après traitement Riplox (12).

Le processus induit donc une forte dénitrification, avec la disparition quasi totale, à l'issue de quelques semaines, de l'apport de nitrate des sédiments et de la colonne d'eau [8]. L'ajout de fer $\left(\mathrm{FeCl}_{3}\right)$ est souvent souhaitable afin d'assurer la bonne fixation ultérieure du phosphore à l'état de $\mathrm{FePO}_{4}$. La mise en œuvre du traitement se fait par passage d'un dispositif d'injection dans la portion de plan d'eau déterminée à l'issue des études préliminaires. Le traitement s'effectue en deux phases immédiatement consécutives: mélange mécanique de la tranche superficielle de sédiment et injection de la solution de nitrate de calcium dans l'horizon $\mathrm{O}-15 \mathrm{~cm}$ (procédé breveté Riplox).

Les résultats du traitement tiennent notamment à la consommation d'oxygène dissous et aux teneurs en $\mathrm{P}_{-} \mathrm{PO}_{4}$ dans la colonne d'eau. La figure 9 présente la demande en oxygène du sédiment avant et après traitement du lac Lillesjoen (Suède) [2]. Cette consommation reste stable 10 ans après le traitement. Le même auteur [9] cite des concentrations de $0,06 \mathrm{mg} / \mathrm{l}$ en phosphore total dans l'eau au-dessus des sédiments, contre 1 à $2 \mathrm{mg} / \mathrm{l}$ avant traitement ; l'eau intersticielle est passée quant à elle de $4-6 \mathrm{mg} / \mathrm{l}$ à $0,4-0,6 \mathrm{mg} / 1$ en $\mathrm{P}_{-} \mathrm{PO}_{4}$ à l'issue du traitement. 


\section{Bibliographie}

[1] Bernhardt H., Clasen J. - Recent developments and perspectives of restoration for artificial basins used for water supply. Proceedings of Lake Pollution and Recovery International Congress, Rome, 1985.

[2] GENEY R. - Improving water quality by reservoir management Waterworld News, A.W.W.A., 1988.

[3] Levet D., Martin X. - Lutte contre l'eutrophisation de la retenue du Cébron par déstratification: Premiers résultats. T.S.M., 1988, 6-88, 339-344.

[4] Allard L. - Note technique. Eutrophisation du réservoir de la Visance. Agence Financière de Bassin Seine-Normandie, Service du Milieu Naturel, 1986.

[5] Fiala L., Vasata P. - Phosphorus reduction in a man-made lake by means of small reservoir on the inflow. Arch. Hydrobiol., 1982, 94-1, 24-37.

[6] Benndorf J., PUTz K. - Control of eutrophication of lakes and reservoirs by means of pre-dams. Water Res., 1987, 21-7, 829-842.

[7] Vymazal J. - The use of periphyton communities for nutrient removal from polluted streams. Hydrobiologia, 1988, $166,225-237$.

[8] Bנork S. - Scandinavian lake restoration activities. Proceedings of Lake Pollution and Recovery International Congress, Rome, 1985.

[9] RIPL W. - Internal phosphorus recycling in shallow lakes. North American Management Society, 1985.

[10] RYDING S.O., RAST W. - The control of eutrophication of lakes and reservoirs. Vol. 1 Man and the biosphere series 115-146. 